Acta Crystallographica Section E

Structure Reports

Online

ISSN 1600-5368

\section{2,4,6-Trimethylpyridinium dihydrogen phosphate}

\section{Hong-Ling Cai* and Jing Dai}

Ordered Matter Science Research Center, College of Chemistry and Chemical Engineering, Southeast University, Nanjing 210096, People's Republic of China Correspondence e-mail: fudavid88@yahoo.com.cn

Received 20 November 2010; accepted 3 December 2010

Key indicators: single-crystal X-ray study; $T=298 \mathrm{~K}$; mean $\sigma(\mathrm{C}-\mathrm{C})=0.005 \AA$; $R$ factor $=0.047 ; w R$ factor $=0.144 ;$ data-to-parameter ratio $=14.3$.

In the title compound, $\mathrm{C}_{8} \mathrm{H}_{9} \mathrm{~N}^{+} \cdot \mathrm{H}_{2} \mathrm{PO}_{4}{ }^{-}$, both the cation and anion have crystallographically imposed mirror symmetry (all atoms apart from one $\mathrm{O}$ atom lie on the mirror plane). In the crystal, anions and cations are linked by $\mathrm{O}-\mathrm{H} \cdots \mathrm{O}$ and $\pi-\pi$ stacking interactions [centroid-centroid distance = 3.4574 (6) $\AA$ ] , forming chains parallel to the $b$ axis. Adjacent chains are further connected by $\mathrm{N}-\mathrm{H} \cdots \mathrm{O}$ hydrogen bonds into a two-dimensional network.

\section{Related literature}

For background to the properties of pyridine salts as phasetransition dielectric materials, see: Fu et al. (2007, 2008, 2009); Fu \& Xiong (2008).

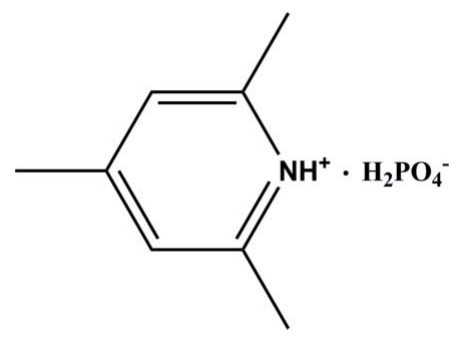

\section{Experimental}

Crystal data

$\mathrm{C}_{8} \mathrm{H}_{9} \mathrm{~N}^{+} \cdot \mathrm{H}_{2} \mathrm{O}_{4} \mathrm{P}^{-} \quad M_{r}=216.15$
Monoclinic, $P 2_{1} / m$

$a=8.6323$ (17)

$b=6.7133$ (13) $\AA$

$c=8.6841(17) \AA$

$\beta=100.99$ (3)

$V=494.02(17) \AA^{3}$

Data collection

Rigaku Mercury2 diffractometer Absorption correction: multi-scan (CrystalClear; Rigaku, 2005)

$T_{\min }=0.910, T_{\max }=1.000$

Refinement

$R\left[F^{2}>2 \sigma\left(F^{2}\right)\right]=0.047$

$w R\left(F^{2}\right)=0.144$

$S=1.18$

1229 reflections

86 parameters

1 restraint
$Z=2$

Mo $K \alpha$ radiation

$\mu=0.27 \mathrm{~mm}^{-1}$

$T=298 \mathrm{~K}$

$0.30 \times 0.05 \times 0.05 \mathrm{~mm}$

5154 measured reflections 1229 independent reflections 1082 reflections with $I>2 \sigma(I)$

$R_{\text {int }}=0.033$

$\mathrm{H}$ atoms treated by a mixture of independent and constrained refinement

$\Delta \rho_{\max }=0.45{\mathrm{e} \AA^{-3}}^{-3}$

$\Delta \rho_{\min }=-0.29 \mathrm{e}^{-3}$

Table 1

Hydrogen-bond geometry $\left(\AA{ }^{\circ}\right)$.

\begin{tabular}{lllll}
\hline$D-\mathrm{H} \cdots A$ & $D-\mathrm{H}$ & $\mathrm{H} \cdots A$ & $D \cdots A$ & $D-\mathrm{H} \cdots A$ \\
\hline $\mathrm{O} 2-\mathrm{H} 2 \cdots \mathrm{O} 3^{\mathrm{i}}$ & $0.85(2)$ & $1.76(2)$ & $2.6054(19)$ & $169(2)$ \\
$\mathrm{N} 1-\mathrm{H} 1 A \cdots \mathrm{O} 1$ & 0.86 & 1.75 & $2.602(3)$ & 173 \\
\hline
\end{tabular}

Symmetry code: (i) $-x,-y,-z+2$.

Data collection: CrystalClear (Rigaku, 2005); cell refinement: CrystalClear; data reduction: CrystalClear; program(s) used to solve structure: SHELXS97 (Sheldrick, 2008); program(s) used to refine structure: SHELXL97 (Sheldrick, 2008); molecular graphics: SHELXTL (Sheldrick, 2008); software used to prepare material for publication: SHELXTL.

This work was supported by a start-up grant from Southeast University, China.

Supplementary data and figures for this paper are available from the IUCr electronic archives (Reference: RZ2528).

\title{
References
}

Fu, D.-W., Ge, J.-Z., Dai, J., Ye, H.-Y. \& Qu, Z.-R. (2009). Inorg. Chem. Commun. 12, 994-997.

Fu, D.-W., Song, Y.-M., Wang, G.-X., Ye, Q., Xiong, R.-G., Akutagawa, T., Nakamura, T., Chan, P. W. H. \& Huang, S.-P.-D. (2007). J. Am. Chem. Soc. 129, 5346-5347.

Fu, D.-W. \& Xiong, R.-G. (2008). Dalton Trans. pp. 3946-3948.

Fu, D.-W., Zhang, W. \& Xiong, R.-G. (2008). Cryst. Growth Des. 8, 3461-3464.

Rigaku (2005). CrystalClear. Rigaku Corporation, Tokyo, Japan.

Sheldrick, G. M. (2008). Acta Cryst. A64, 112-122. 


\section{supporting information}

Acta Cryst. (2011). E67, o72 [https://doi.org/10.1107/S1600536810050646]

\section{2,4,6-Trimethylpyridinium dihydrogen phosphate}

\section{Hong-Ling Cai and Jing Dai}

\section{S1. Comment}

Salts of pyridine have attracted attention as phase transition dielectric materials for their applications in memory storage (Fu et al. 2007; Fu \& Xiong 2008; Fu et al. 2008; Fu et al. 2009). With the purpose of obtaining new phase transition crystals of 2,4,6-trimethylpyridine salts, their interaction with various acids has been studied and we have elaborated a series of new materials with this organic molecule. In this study, we describe the crystal structure of the title compound, 2,4,6-trimethylpyridinium dihydrogen phosphate.

The asymmetric unit is composed of half an $\mathrm{H}_{2} \mathrm{PO}_{4}^{-}$anion and half $\mathrm{C}_{8} \mathrm{H}_{9} \mathrm{~N}^{+}$cation (Fig. 1), both anion and cation being located on a mirror plane. The geometric parameters are in the normal range. In the crystal structure, the anions are linked into chains parallel to the $b$ axis by $\mathrm{O}-\mathrm{H} \cdots \mathrm{O}$ hydrogen bonds (Table 1). The cations also are connected into chains along the $b$ axis by $\pi-\pi$ stacking interactions with centroid-to-centroid distances of 3.4574 (6) $\AA$. The cationic and anionic chains further interact through $\mathrm{N}-\mathrm{H} \cdots \mathrm{O}$ hydrogen bonds (Fig. 2), forming a two-dimensional network.

\section{S2. Experimental}

The commercial 2,4,6-trimethylpyridine (3 mmol) was dissolved in water $/ \mathrm{H}_{3} \mathrm{PO}_{4}(50: 1 \mathrm{v} / \mathrm{v})$ solution. The solvent was slowly evaporated in air affording colourless block-shaped crystals of the title compound suitable for X-ray analysis.

The dielectric constant of title compound as a function of temperature indicates that the permittivity is basically temperature-independent, suggesting that this compound should be not a real ferroelectrics or there may be no distinct phase transition occurred within the measured temperature range. Similarly, below the melting point (413 K) of the compound, the dielectric constant as a function of temperature also goes smoothly, and there is no dielectric anomaly observed (dielectric constant equaling to 6.6 to 8.9 ).

\section{S3. Refinement}

All $\mathrm{H}$ atoms attached to $\mathrm{C}$ and $\mathrm{N}$ atoms were fixed geometrically and treated as riding with $\mathrm{C}-\mathrm{H}=0.93-0.96 \AA, \mathrm{N}-\mathrm{H}=$ $0.86 \AA$, and with $U_{\text {iso }}(\mathrm{H})=1.2 U_{\text {eq }}(\mathrm{C}, \mathrm{N})$ or $1.5 U_{\text {eq }}(\mathrm{C})$ for methyl $\mathrm{H}$ atoms. The $\mathrm{H}$ atom of the $\mathrm{H}_{2} \mathrm{PO}_{4}^{-}$anion was located in difference Fourier maps and freely refined, with the $\mathrm{O}-\mathrm{H}$ distance constrained to $0.86 \AA$.. 


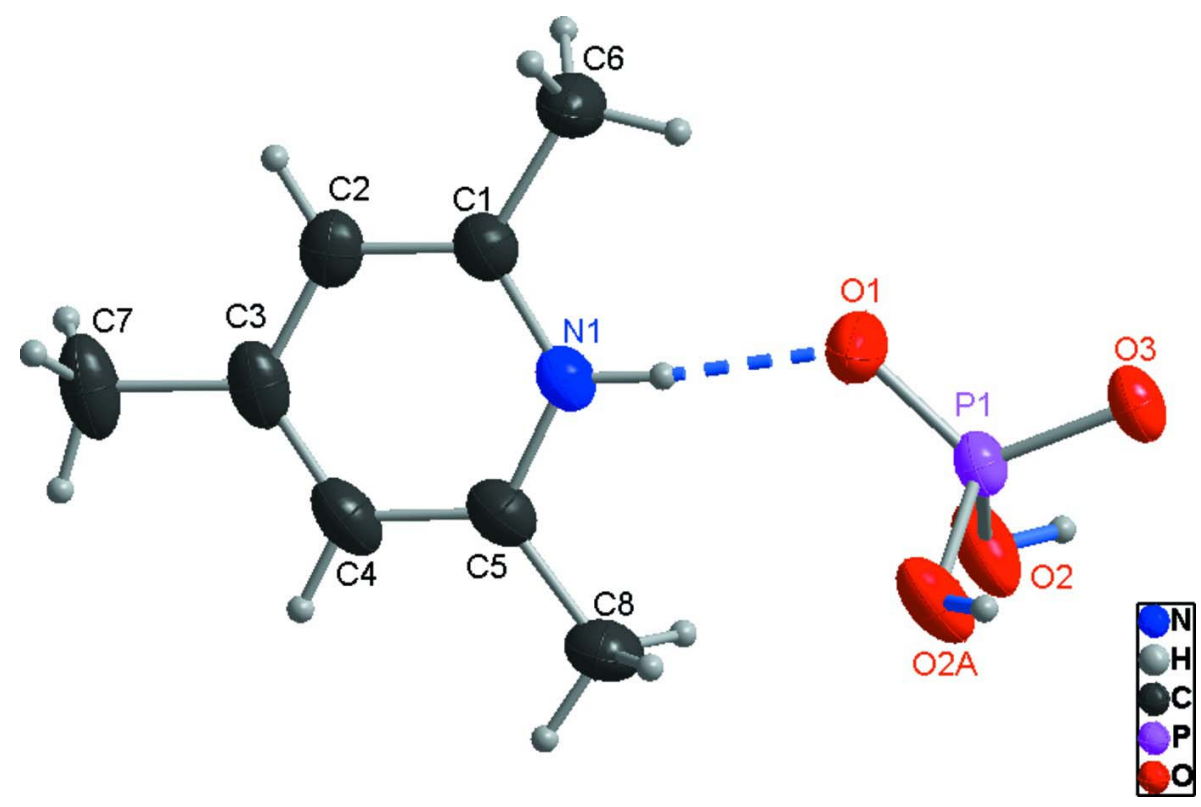

Figure 1

A view of the title compound with the atomic numbering scheme. Displacement ellipsoids were drawn at the $30 \%$ probability level. Symmetry code: (A) x, 0.5-y, z. 


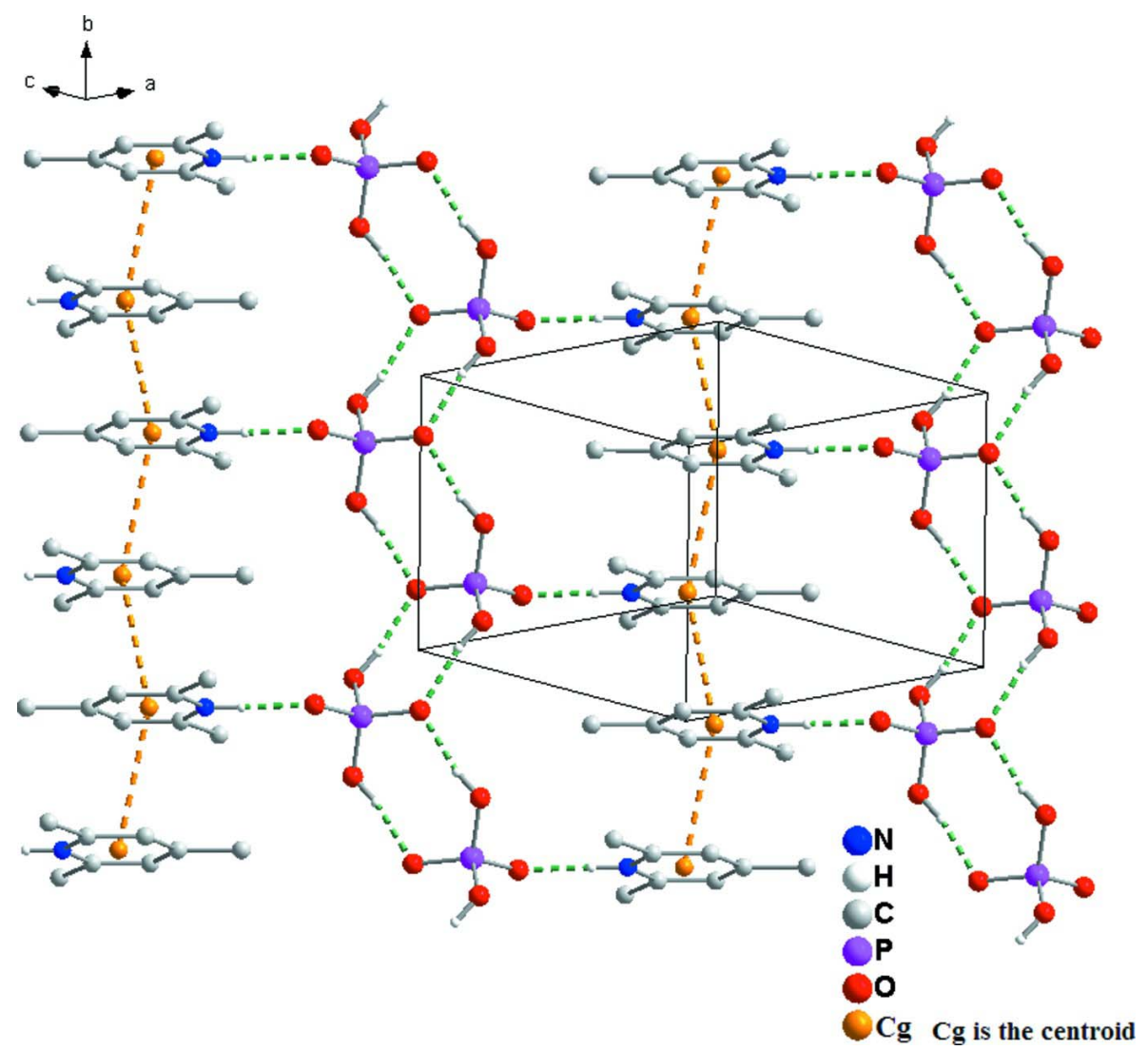

Figure 2

The crystal packing of the title compound, showing the two-dimensional network. $\mathrm{H}$ atoms not involved in hydrogen bonding (dashed line) have been omitted for clarity.

\section{2,4,6-Trimethylpyridinium dihydrogen phosphate}

\section{Crystal data}

$\mathrm{C}_{8} \mathrm{H}_{9} \mathrm{~N}^{+} \cdot \mathrm{H}_{2} \mathrm{O}_{4} \mathrm{P}^{-}$

$M_{r}=216.15$

Monoclinic, $P 2_{1} / m$

Hall symbol: -P $2 \mathrm{yb}$

$a=8.6323(17) \AA$

$b=6.7133(13) \AA$

$c=8.6841$ (17) $\AA$

$\beta=100.99(3)^{\circ}$

$V=494.02(17) \AA^{3}$

$Z=2$

\section{Data collection}

\section{Rigaku Mercury2}

diffractometer

Radiation source: fine-focus sealed tube Graphite monochromator

Detector resolution: 13.6612 pixels $\mathrm{mm}^{-1}$

CCD profile fitting scans
$F(000)=226$

$D_{\mathrm{x}}=1.453 \mathrm{Mg} \mathrm{m}^{-3}$

Mo $K \alpha$ radiation, $\lambda=0.71073 \AA$

Cell parameters from 1229 reflections

$\theta=3.1-27.5^{\circ}$

$\mu=0.27 \mathrm{~mm}^{-1}$

$T=298 \mathrm{~K}$

Block, colorless

$0.30 \times 0.05 \times 0.05 \mathrm{~mm}$

Absorption correction: multi-scan

(CrystalClear; Rigaku, 2005)

$T_{\min }=0.910, T_{\max }=1.000$

5154 measured reflections

1229 independent reflections

1082 reflections with $I>2 \sigma(I)$

$R_{\text {int }}=0.033$ 
$\theta_{\max }=27.5^{\circ}, \theta_{\min }=3.1^{\circ}$

$h=-11 \rightarrow 11$

\section{Refinement}

Refinement on $F^{2}$

Least-squares matrix: full

$R\left[F^{2}>2 \sigma\left(F^{2}\right)\right]=0.047$

$w R\left(F^{2}\right)=0.144$

$S=1.18$

1229 reflections

86 parameters

1 restraint

Primary atom site location: structure-invariant direct methods $k=-8 \rightarrow 8$

$l=-11 \rightarrow 11$

Secondary atom site location: difference Fourier map

Hydrogen site location: inferred from neighbouring sites

$\mathrm{H}$ atoms treated by a mixture of independent and constrained refinement

$w=1 /\left[\sigma^{2}\left(F_{\mathrm{o}}^{2}\right)+(0.0788 P)^{2}+0.1875 P\right]$ where $P=\left(F_{\mathrm{o}}^{2}+2 F_{\mathrm{c}}^{2}\right) / 3$

$(\Delta / \sigma)_{\max }<0.001$

$\Delta \rho_{\max }=0.45$ e $\AA^{-3}$

$\Delta \rho_{\min }=-0.29$ e $\AA^{-3}$

\section{Special details}

Geometry. All e.s.d.'s (except the e.s.d. in the dihedral angle between two 1.s. planes) are estimated using the full covariance matrix. The cell e.s.d.'s are taken into account individually in the estimation of e.s.d.'s in distances, angles and torsion angles; correlations between e.s.d.'s in cell parameters are only used when they are defined by crystal symmetry. An approximate (isotropic) treatment of cell e.s.d.'s is used for estimating e.s.d.'s involving 1.s. planes.

Refinement. Refinement of $F^{2}$ against ALL reflections. The weighted $R$-factor $w R$ and goodness of fit $S$ are based on $F^{2}$, conventional $R$-factors $R$ are based on $F$, with $F$ set to zero for negative $F^{2}$. The threshold expression of $F^{2}>\sigma\left(F^{2}\right)$ is used only for calculating $R$-factors(gt) etc. and is not relevant to the choice of reflections for refinement. $R$-factors based on $F^{2}$ are statistically about twice as large as those based on $F$, and $R$ - factors based on ALL data will be even larger.

Fractional atomic coordinates and isotropic or equivalent isotropic displacement parameters $\left(\AA^{2}\right)$

\begin{tabular}{llllll}
\hline & $x$ & $y$ & $z$ & $U_{\text {iso }} * U_{\text {eq }}$ & Occ. $(<1)$ \\
\hline N1 & $0.3386(3)$ & 0.2500 & $0.5841(3)$ & $0.0298(5)$ & \\
H1A & 0.2650 & 0.2500 & 0.6385 & $0.036^{*}$ & \\
C3 & $0.5712(4)$ & 0.2500 & $0.4112(4)$ & $0.0382(7)$ & \\
C2 & $0.4135(4)$ & 0.2500 & $0.3392(4)$ & $0.0378(7)$ & \\
H2A & 0.3862 & 0.2500 & 0.2303 & $0.045^{*}$ & \\
C1 & $0.2974(3)$ & 0.2500 & $0.4270(3)$ & $0.0333(6)$ & \\
C5 & $0.4890(3)$ & 0.2500 & $0.6600(4)$ & $0.0338(6)$ & \\
C4 & $0.6066(3)$ & 0.2500 & $0.5731(4)$ & $0.0383(7)$ & \\
H4A & 0.7115 & 0.2500 & 0.6242 & $0.046^{*}$ & \\
C7 & $0.6980(5)$ & 0.2500 & $0.3145(5)$ & $0.0548(10)$ & \\
H7A & 0.7986 & 0.2500 & 0.3845 & $0.082^{*}$ & \\
H7B & 0.6891 & 0.1332 & 0.2496 & $0.082^{*}$ & \\
C8 & $0.5179(4)$ & 0.2500 & $0.8351(4)$ & $0.0456(8)$ & \\
H8A & 0.6296 & 0.2500 & 0.8750 & $0.068^{*}$ & \\
H8B & 0.4717 & 0.3668 & 0.8715 & $0.068^{*}$ & \\
C6 & $0.1257(4)$ & 0.2500 & $0.3578(4)$ & $0.0505(9)$ & \\
H6A & 0.0675 & 0.2500 & 0.4417 & $0.076^{*}$ & \\
H6B & 0.0992 & 0.3668 & 0.2945 & $0.076^{*}$ & \\
P1 & $0.09606(8)$ & 0.2500 & $0.89656(8)$ & $0.0280(3)$ & \\
O1 & $0.0994(3)$ & 0.2500 & $0.7269(3)$ & $0.0515(7)$ & \\
O2 & $0.19545(18)$ & $0.0720(3)$ & $0.9782(2)$ & $0.0494(5)$ & \\
O3 & $-0.0654(2)$ & 0.2500 & $0.9371(2)$ & $0.0343(5)$ & \\
& & & & &
\end{tabular}




$\begin{array}{lllll}\mathrm{H} 2 & 0.145(3) & -0.024(3) & 1.010(3) & 0.066(9)^{*}\end{array}$

Atomic displacement parameters $\left(\AA^{2}\right)$

\begin{tabular}{lllllll}
\hline & $U^{11}$ & $U^{22}$ & $U^{33}$ & $U^{12}$ & $U^{13}$ & $U^{23}$ \\
\hline $\mathrm{N} 1$ & $0.0261(11)$ & $0.0273(11)$ & $0.0368(12)$ & 0.000 & $0.0079(9)$ & 0.000 \\
$\mathrm{C} 3$ & $0.0383(16)$ & $0.0230(13)$ & $0.0587(19)$ & 0.000 & $0.0226(14)$ & 0.000 \\
$\mathrm{C} 2$ & $0.0431(17)$ & $0.0325(15)$ & $0.0408(16)$ & 0.000 & $0.0156(13)$ & 0.000 \\
$\mathrm{C} 1$ & $0.0325(14)$ & $0.0303(14)$ & $0.0375(15)$ & 0.000 & $0.0078(12)$ & 0.000 \\
$\mathrm{C} 5$ & $0.0289(14)$ & $0.0270(13)$ & $0.0446(16)$ & 0.000 & $0.0044(12)$ & 0.000 \\
$\mathrm{C} 4$ & $0.0256(13)$ & $0.0288(14)$ & $0.0609(19)$ & 0.000 & $0.0091(13)$ & 0.000 \\
$\mathrm{C} 7$ & $0.0469(19)$ & $0.0458(19)$ & $0.082(3)$ & 0.000 & $0.0385(19)$ & 0.000 \\
C8 & $0.0344(16)$ & $0.056(2)$ & $0.0419(17)$ & 0.000 & $-0.0032(13)$ & 0.000 \\
C6 & $0.0344(16)$ & $0.080(3)$ & $0.0366(16)$ & 0.000 & $0.0040(13)$ & 0.000 \\
P1 & $0.0243(4)$ & $0.0276(4)$ & $0.0340(4)$ & 0.000 & $0.0102(3)$ & 0.000 \\
O1 & $0.0383(12)$ & $0.0835(19)$ & $0.0353(12)$ & 0.000 & $0.0131(9)$ & 0.000 \\
O2 & $0.0297(8)$ & $0.0390(9)$ & $0.0826(13)$ & $0.0050(7)$ & $0.0181(8)$ & $0.0211(8)$ \\
O3 & $0.0267(10)$ & $0.0300(10)$ & $0.0493(12)$ & 0.000 & $0.0152(9)$ & 0.000 \\
& & & & & & \\
\hline
\end{tabular}

Geometric parameters $\left(A,{ }^{\circ}\right)$

\begin{tabular}{llll}
\hline $\mathrm{N} 1-\mathrm{C} 5$ & $1.339(4)$ & $\mathrm{C} 7-\mathrm{H} 7 \mathrm{~A}$ & 0.9601 \\
$\mathrm{~N} 1-\mathrm{C} 1$ & $1.343(4)$ & $\mathrm{C} 7-\mathrm{H} 7 \mathrm{~B}$ & 0.9600 \\
$\mathrm{~N} 1-\mathrm{H} 1 \mathrm{~A}$ & 0.8600 & $\mathrm{C} 8-\mathrm{H} 8 \mathrm{~A}$ & 0.9600 \\
$\mathrm{C} 3-\mathrm{C} 4$ & $1.381(5)$ & $\mathrm{C} 8-\mathrm{H} 8 \mathrm{~B}$ & 0.9600 \\
$\mathrm{C} 3-\mathrm{C} 2$ & $1.385(5)$ & $\mathrm{C} 6-\mathrm{H} 6 \mathrm{~A}$ & 0.9600 \\
$\mathrm{C} 3-\mathrm{C} 7$ & $1.501(4)$ & $\mathrm{C} 6-\mathrm{H} 6 \mathrm{~B}$ & 0.9600 \\
$\mathrm{C} 2-\mathrm{C} 1$ & $1.371(4)$ & $\mathrm{P} 1-\mathrm{O} 1$ & $1.479(2)$ \\
$\mathrm{C} 2-\mathrm{H} 2 \mathrm{~A}$ & 0.9300 & $\mathrm{P} 1-\mathrm{O} 3$ & $1.502(2)$ \\
$\mathrm{C} 1-\mathrm{C} 6$ & $1.489(4)$ & $\mathrm{P} 1-\mathrm{O} 2^{\mathrm{i}}$ & $1.5603(17)$ \\
$\mathrm{C} 5-\mathrm{C} 4$ & $1.376(4)$ & $\mathrm{P} 1-\mathrm{O} 2$ & $1.5603(16)$ \\
$\mathrm{C} 5-\mathrm{C} 8$ & $1.493(4)$ & $\mathrm{O} 2-\mathrm{H} 2$ & $0.85(2)$ \\
$\mathrm{C} 4-\mathrm{H} 4 \mathrm{~A}$ & 0.9300 & & 119.5 \\
& & & 108.3 \\
$\mathrm{C} 5-\mathrm{N} 1-\mathrm{C} 1$ & $123.0(3)$ & $\mathrm{C} 3-\mathrm{C} 4-\mathrm{H} 4 \mathrm{~A}$ & 110.1 \\
$\mathrm{C} 5-\mathrm{N} 1-\mathrm{H} 1 \mathrm{~A}$ & 118.5 & $\mathrm{C} 3-\mathrm{C} 7-\mathrm{H} 7 \mathrm{~A}$ & 109.5 \\
$\mathrm{C} 1-\mathrm{N} 1-\mathrm{H} 1 \mathrm{~A}$ & 118.5 & $\mathrm{C} 3-\mathrm{C} 7-\mathrm{H} 7 \mathrm{~B}$ & 109.2 \\
$\mathrm{C} 4-\mathrm{C} 3-\mathrm{C} 2$ & $117.9(3)$ & $\mathrm{H} 7 \mathrm{~A}-\mathrm{C} 7-\mathrm{H} 7 \mathrm{~B}$ & 109.6 \\
$\mathrm{C} 4-\mathrm{C} 3-\mathrm{C} 7$ & $121.7(3)$ & $\mathrm{C} 5-\mathrm{C} 8-\mathrm{H} 8 \mathrm{~A}$ & 109.5 \\
$\mathrm{C} 2-\mathrm{C} 3-\mathrm{C} 7$ & $120.4(3)$ & $\mathrm{C} 5-\mathrm{C} 8-\mathrm{H} 8 \mathrm{~B}$ & 108.5 \\
$\mathrm{C} 1-\mathrm{C} 2-\mathrm{C} 3$ & $120.6(3)$ & $\mathrm{H} 8 \mathrm{~A}-\mathrm{C} 8-\mathrm{H} 8 \mathrm{~B}$ & 109.9 \\
$\mathrm{C} 1-\mathrm{C} 2-\mathrm{H} 2 \mathrm{~A}$ & 119.7 & $\mathrm{C} 1-\mathrm{C} 6-\mathrm{H} 6 \mathrm{~A}$ & 109.5 \\
$\mathrm{C} 3-\mathrm{C} 2-\mathrm{H} 2 \mathrm{~A}$ & 119.7 & $\mathrm{C} 1-\mathrm{C} 6-\mathrm{H} 6 \mathrm{~B}$ & $115.41(13)$ \\
$\mathrm{N} 1-\mathrm{C} 1-\mathrm{C} 2$ & $119.0(3)$ & $\mathrm{H} 6 \mathrm{~A}-\mathrm{C} 6-\mathrm{H} 6 \mathrm{~B}$ & $109.81(9)$ \\
$\mathrm{N} 1-\mathrm{C} 1-\mathrm{C} 6$ & $117.4(3)$ & $\mathrm{O} 1-\mathrm{P} 1-\mathrm{O} 3$ & $110.37(8)$ \\
$\mathrm{C} 2-\mathrm{C} 1-\mathrm{C} 6$ & $123.5(3)$ & $\mathrm{O} 1-\mathrm{P} 1-\mathrm{O} 2 \mathrm{i}$ & $109.81(9)$ \\
$\mathrm{N} 1-\mathrm{C} 5-\mathrm{C} 4$ & $118.5(3)$ & $\mathrm{O} 1-\mathrm{P} 1-\mathrm{O} 2$ & \\
$\mathrm{~N} 1-\mathrm{C} 5-\mathrm{C} 8$ & $117.4(3)$ & & \\
& & &
\end{tabular}




$\begin{array}{llll}\mathrm{C} 4-\mathrm{C} 5-\mathrm{C} 8 & 124.1(3) & \mathrm{O} 3-\mathrm{P} 1-\mathrm{O} 2 & 110.37(8) \\ \mathrm{C} 5-\mathrm{C} 4-\mathrm{C} 3 & 121.1(3) & \mathrm{O} 2-\mathrm{i} 1-\mathrm{O} 2 & 99.97(14) \\ \mathrm{C} 5-\mathrm{C} 4-\mathrm{H} 4 \mathrm{~A} & 119.5 & \mathrm{P} 1-\mathrm{O} 2-\mathrm{H} 2 & 117(2)\end{array}$

Symmetry code: (i) $x,-y+1 / 2, z$.

Hydrogen-bond geometry $\left(\AA,{ }^{\circ}\right)$

\begin{tabular}{lllll}
\hline$D-\mathrm{H} \cdots A$ & $D-\mathrm{H}$ & $\mathrm{H} \cdots A$ & $D \cdots A$ & $D-\mathrm{H} \cdots A$ \\
\hline $\mathrm{O} 2-\mathrm{H} 2 \cdots \mathrm{O} 3^{\mathrm{ii}}$ & $0.85(2)$ & $1.76(2)$ & $2.6054(19)$ & $169(2)$ \\
$\mathrm{N} 1-\mathrm{H} 1 A \cdots \mathrm{O} 1$ & 0.86 & 1.75 & $2.602(3)$ & 173 \\
\hline
\end{tabular}

Symmetry code: (ii) $-x,-y,-z+2$. 